\title{
Using Multi-Element Reference Materials for Approximating Concentrations in Soils and Sediments from Micro XRF Spectra
}

Stewart McIntyre, Lisa Van Loon, Nathaniel Sherry, Michael Bauer and Neil Banerjee

Western University, London, Ontario, Canada

\section{Introduction}

This paper reports a rapid and intuitive treatment process for $X$ ray fluorescence (XRF) data to determine elemental concentrations in low $\mathrm{Z}$ siliceous materials. To estimate the concentration of an element in an unknown material, a reference material is required with a matrix whose composition is similar to that of the unknown. NIST SRM 610, a siliceous glass matrix containing as many as 60 elements with certified concentrations in the $300-/ 500 \mathrm{mg} / \mathrm{kg}$ range [1], has been widely used as a primary standard in geochemistry and environmental research. Importantly for microanalysis, the composition is homogeneous for the majority of elements [2]. We have developed a protocol using an intuitive XRF analysis software, Peakaboo [3] for the rapid but exacting computational fitting of a very complex XRF spectrum to provide a rapid multi-element quantitative assay. To test the accuracy, we determined the elemental concentration ratios in NIST SRM 2711a (Montana Soil) [4] using NIST SRM 610 as a primary reference.

\section{Experimental}

NIST SRM 610 is a silicate glass with 61 elements added at the $\sim 450 \mathrm{mg} / \mathrm{kg}$ level. The concentrations of 24 elements are either certified or referenced. NIST SRM 2711a is a finely milled and dried agricultural soil from a locale near a smelting plant. Homogeneity is better than $1 \%$. Twenty-seven minor or trace elements are certified or referenced, while others are provided for information.

Synchrotron radiation XRF (SR-XRF) data was collected at the APS at Beamline 20-ID [5]. The incident energy was $26 \mathrm{keV}$. Data was collected in fluorescence mode using a single element Vortex SDD. For SRM 610 , areas of $6000 \mu \mathrm{m} \times 6000 \mu \mathrm{m}$ were mapped with a $500 \mu \mathrm{m}$ × $500 \mu \mathrm{m}$ spot size (169 data points) and a 500 $\mu \mathrm{sec}$ dwell time. The averaged spectrum was used. For SRM 2711a, three separate 60s exposures were taken in three locations on the sample using a $500 \mu \mathrm{m}$ x $500 \mu \mathrm{m}$ spot size. The three measurements were analyzed, and the results were found to be comparable.

Peakaboo [3] provides an easy-to-use approach to interpreting all features in an XRF spectrum and rapidly presents megapixel data sets as understandable 2D element maps. Figure 1 shows the fitting of the NIST SRM 610 spectrum from $7 \mathrm{keV}$ to $12 \mathrm{keV}$ showing (a) peak envelopes and (b) the individual XRF transitions for each element. At energies below $12 \mathrm{keV}$ the entire spectrum can be fit with the contributions from the elements present. At energies above $12 \mathrm{keV}$ background contributions appear to be present.

The quantification process of the elements in SRM 2711a requires that both the unknown and a reference material be analyzed under the same condition and their spectra be subject to identical fitting conditions. Quantification of the unknown spectra is then conducted as follows:

The summed peak intensities (Ielement) are ratioed to the summed intensities of the Fe $\mathrm{K}_{\alpha}$ and Fe $\mathrm{K}_{\beta}$ Here, the $\mathrm{Fe}$ intensity is used as a reference because $\mathrm{Fe}$ is present in all materials analyzed.

$$
\text { Ratioed Peak Intensity }=\frac{\text { Summed Peak Intensities }}{\text { Element }}
$$


The XRF yield for each element is determined using the certified or reference concentrations

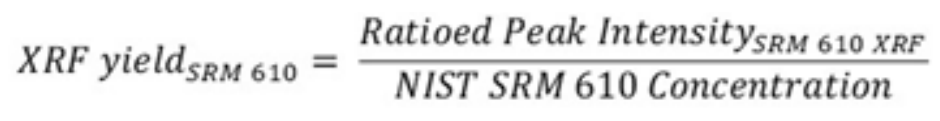

The element concentration ratios for the unknown sample are then calculated.

$$
\text { Concentration Ratio }_{\text {element }}=\frac{\text { Ratioed Peak Intensity }_{\text {element }}}{X R F \text { yield }}
$$

The accuracy of the Concentration Ratio with respect to the ratio from the published NIST values is expressed as the Error Factor (EF). The Error Factor (EF) is always greater than one.

or

$$
\text { Error Factor }=\frac{{\text { Concentration } \text { Ratio }_{\text {element }}}}{\left(\frac{[\text { element }]}{[\mathrm{Fe}]}\right)}
$$

$$
\frac{\left(\frac{[\text { element }]}{[\mathrm{Fe}]}\right)}{\text { Concentration Ratio }_{\text {element }}} \text { so Error Factor } \geq 1
$$

\section{Results and Discussion}

NIST SRM 2711a is treated here as an "unknown" sample, to compare the results from our quantification process with the ratios from the certified and reference analysis data for NIST SRM 2711a. Data for elements below $\mathrm{Ca}$ in the periodic table are not presented because of low sensitivity of the detector to elements in that range.

The intense Fe $\mathrm{K}$ lines in the NIST SRM 2711a spectrum overshadow the $\mathrm{K}$ and L lines of neighboring elements. ( $\mathrm{Fe}=2.82 \mathrm{mass} \%, \mathrm{Mn}=675 \mathrm{mg} / \mathrm{kg}$, and $\mathrm{Ni}=21.7 \mathrm{mg} / \mathrm{kg}$ ). Thus, the accuracy of measurements for neighboring elements is considered a test of the fitting accuracy, as well as the assumptions that secondary fluorescence and absorption effects are minor. The analysis of closely-spaced peak shapes requires considerable care so that overlapping peaks do not interfere. The experimental concentration ratio for each element was determined from an average of four separate quantification determinations. The error factors (EF) for the quantification process varied from 1 (perfect agreement) to 10 (a concentration ratio 10 times away from the NIST value). The mean error factor for all 22 elements measured was 2.5. The results for elements with peaks in the energy range $4.5 \mathrm{keV}-12 \mathrm{keV}\left(\mathrm{EF}_{\mathrm{avg}}=1.5\right)$ are most accurate. The results for $\mathrm{Mn}$ and $\mathrm{Ni}$ and $\mathrm{Co}(\mathrm{EF}=1-2)$ are encouraging since their peaks are near the intense Fe K peaks. The measurement of $\mathrm{X}$ ray yields for elements at lower energies is less accurate $(\mathrm{EF}>2)$ possibly because of poor detector resolution leading to inaccurate XRF yields. Elements with $\mathrm{K}$ lines above $12 \mathrm{keV}$ also have a higher error factor $(\mathrm{EF} \geq 4)$ which may be due to differing background contributions above $12 \mathrm{keV}$ for the two SRM's. Despite the low concentrations of many of the elements detected by their L lines, the accuracy of their detection proved to be encouraging. This analysis protocol could form the basis for rapid automated analysis and for large area XRF mapping applications. 


\section{Acknowledgement}

This research used resources of the Advanced Photon Source, an Office of Science User Facility operated for the U.S. Department of Energy (DOE) Office of Science by Argonne National Laboratory, and was supported by the U.S. DOE under Contract No. DE-AC02-06CH11357, and the Canadian Light Source and its funding partners. We thank Dr. Z. Finfrock and the APS HSE staff for support in conducting the experiments. The authors acknowledge funding from NSERC and CANARIE.

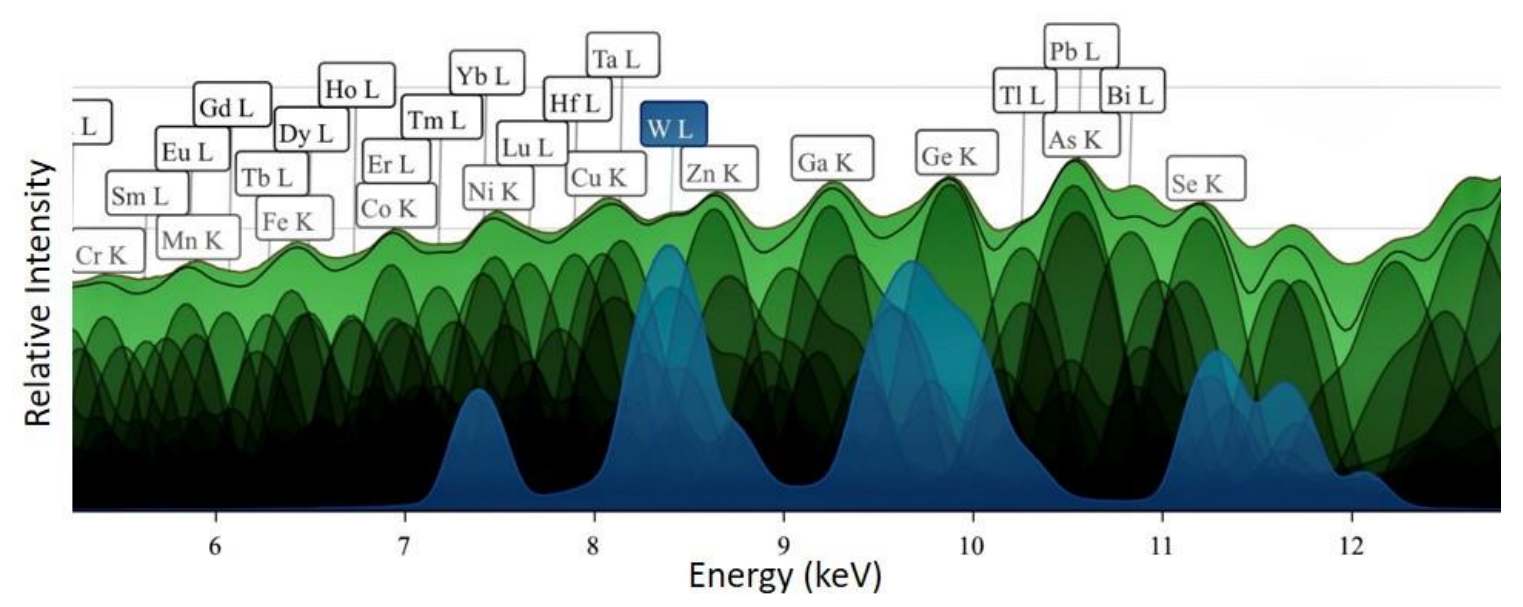

Figure 1. A portion of the XRF spectrum of NIST SRM 610 showing the fitted contributions for K and L peaks. The intensities are presented on a logarithmic scale with the maximum intensity near 4200 counts. The spectral energy is given in keV. The collected XRF spectrum is shown in light green. The element peak fittings are shown in dark green. The contributions from the $7 \mathrm{~W} \mathrm{~L}$ peaks are shown in blue. The overall fit is shown as a black line.

\section{References}

[1] Wise, S. A. \& Watters Jr., R. L. (2012). Certificate of Analysis, Standard Reference Material® 610 - Trace Elements in Glass.

[2] Jochum, K. P., Weis, U., Stoll, B., Kuzmin, D., Yang, Q., Raczek, I., Jacob, D. E., Stracke, A., Birbaum, K., Frick, D. A., Günther, D. \& Enzweiler, J. (2011). Geostand. Geoanalytical Res. 35, 397-429.

[3] Van Loon, L. L., McIntyre, N. S., Bauer, M., Sherry, N. S. A. \& Banerjee, N. R. (2019). Softw. Impacts. 2 , 100010.

[4] Mackey, E. A. \& et al (2010). Certification of Three NIST Renewal Soil Standard Reference Materials: SRM 2709a San Joachim Soil, SRM 2710a Montana Soil I and SRM 2711a Montana Soil II.

[5] Heald, S., Stern, E., Brewe, D., Gordon, R., Crozier, D., Jiang, D. \& Cross, J. (2001). J. Synchrotron Radiat. 8, 342-344. 